\title{
DESARROLLO URBANO E INUNDACIONES EN LA CIUDAD DE LAS PALMAS DE GRAN CANARIA (1869-2000) ${ }^{1}$
}

\author{
Pablo Máyer Suárez \\ Departamento de Geografía, Área de Geografía Física. \\ Universidad de Las Palmas de Gran Canaria
}

\section{RESUMEN}

En la ciudad de Las Palmas de Gran Canaria los episodios de lluvia facilitan la manifestación de riesgos geomorfológicos. El de las inundaciones constituye uno de los más importantes y ha estado presente desde los orígenes de esta ciudad. Las peculiares características del medio físico, tanto desde el punto de vista topográfico como desde el geológico-geomorfológico; las distintas formas de ocupación del espacio, con áreas de crecimiento planificadas y otras de crecimiento espontáneo, y las diversas intervenciones en áreas consolidadas, que potencian o agravan estas inundaciones, hacen de esta ciudad una amalgama en la que el análisis de las causas de las inundaciones resulta un proceso complejo.

Palabras clave: Las Palmas de Gran Canaria, inundaciones, daños, precipitaciones intensas.

\begin{abstract}
Incidents of heavy rainfall in the city of Las Palmas de Gran Canaria pose various geomorphologic risks. One of the most important of these is flooding- a risk that has existed from the very time the city was first built. Topographical and geological-geomorphologic peculiarities of the physical environment; various forms of land occupation with some areas of development being planned whilst others come into being in a spontaneous manner and; various interventions that serve to strengthen or worsen these floods in consolidated areas together make this city a complex structure in which analysis of the causes of flooding is a complex process.
\end{abstract}

Key words: Las Palmas de Gran Canaria, flood, damages, hevy rainfall.

1 Este trabajo ha sido realizado gracias a la concesión de una Beca de Investigación y Formación del Profesorado por la Universidad de Las Palmas de Gran Canaria en octubre de 1997. 


\section{Introducción}

La planificación territorial exige el desarrollo de estudios orientados al conocimiento de diferentes riesgos naturales y antrópicos. Desde el punto de vista conceptual, el riesgo comporta dos subsistemas en interacción: la sociedad que es afectada y el proceso natural desencadenante. A estos dos subsistemas se añade, por una parte, la dimensión espacial del proceso natural según la localización de las actividades antrópicas y, por otra, el parámetro temporal de ocurrencia del fenómeno, que convierte en real lo que en potencia era un riesgo (Ortega, 1991: 102).

El concepto de riesgo lleva aparejado también el de incertidumbre. Su prevención hace necesario conocer el funcionamiento de los procesos naturales, su intensidad, y su frecuencia o recurrencia en el tiempo; pero es necesario analizar todo ello de forma aplicada, en tanto que el proceso natural actúa sobre un grupo humano. En este sentido, la ciudad de Las Palmas de Gran Canaria es un escenario donde la interacción de los procesos naturales junto con el rápido crecimiento urbano, especialmente a partir de la segunda mitad del siglo $\mathrm{XX}$, ha dado lugar a la aparición de numerosos riesgos, la mayoría de ellos inducidos. Prueba de ello son las numerosas crónicas de historiadores, así como de algunos ciudadanos, que plasmaron en sus escritos las inundaciones padecidas por la ciudad como consecuencia de precipitaciones intensas. Con la publicación de los primeros periódicos locales, las referencias de inundaciones y otros daños con motivo de los temporales que afectan a la isla comienzan a ser recurrentes, de tal forma que los problemas, aún siendo los mismos, alcanzan áreas cada vez más extensas. A partir de la segunda mitad del siglo XX, las noticias periodísticas que aluden a los problemas ocasionados en la ciudad, ya sea por fuertes aguaceros o por lluvias moderadas, tienen un correlato en la prensa cada vez más frecuente. Se pone así de manifiesto una problemática singular que ha padecido la ciudad a lo largo de su historia, problemática analizada en esta investigación a partir del análisis de las consecuencias de las lluvias registradas en la ciudad en los últimos 132 años.

\section{Objetivos}

El principal objetivo de esta investigación es analizar las consecuencias ambientales de las lluvias intensas en la ciudad de Las Palmas de Gran Canaria desde 1869 hasta el año 2000, así como la variación espacial de sus efectos en función de la evolución de la trama urbana.

Este objetivo general se desglosa en los siguientes objetivos específicos: analizar cuáles han sido las áreas que han sufrido inundaciones a lo largo del período considerado, establecer las causas de origen antrópico, así como su relación con las grandes fases de crecimiento urbano; determinar los umbrales de precipitación a partir de los cuáles se desencadenan problemas y daños en la ciudad; y, finalmente, analizar las consecuencias socioeconómicas derivadas de los episodios de lluvia intensa, con especial referencia a las víctimas y daños producidos en los distintos sectores de actividad.

\section{3. Área de estudio}

El entorno sobre el que se desarrolla la evolución urbana de Las Palmas de Gran Canaria se sitúa en el NE de la isla de Gran Canaria. El primitivo núcleo de población se asentó en el margen derecho del Barranco Guiniguada, muy cerca de su desembocadura. Con el 
transcurso de los años, distintas actuaciones en el medio, que ofrecía abundantes recursos para la sociedad del momento, permitieron la expansión de la ciudad por el territorio próximo.

En este espacio se pueden diferenciar distintas unidades de paisaje, cuyo origen habría que buscarlo en los millones de años que tardó en configurarse el soporte físico y en los escasos siglos que se tardó en modificarlo y adaptarlo, cuando esto fue posible, a las necesidades del grupo humano que se asentó sobre él (figura 1).

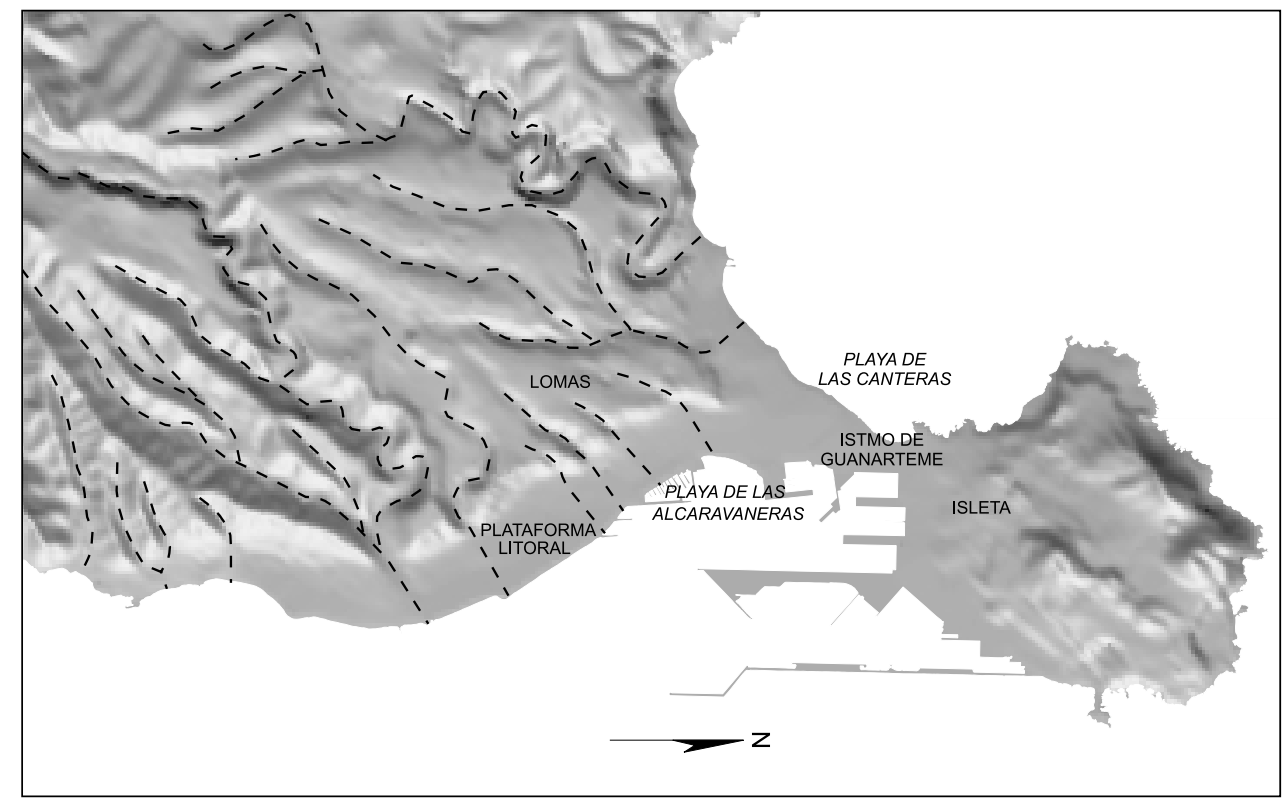

FIgURA 1. Croquis de distintos sectores de Las Palmas de Gran Canaria.

Entre las unidades que favorecieron el asentamiento urbano, destaca la existencia de una estrecha franja litoral que se extiende entre el mar, hacia naciente, y las lomas y riscos, hacia poniente, con un desarrollo longitudinal que tiene como límite Sur la playa de La Laja, y como límite Norte el istmo de Guanarteme. Se trata de una plataforma de abrasión marina sobre la que se depositaron sedimentos coluviales, procedentes del desmantelamiento del acantilado marino fósil que lo circunda, y sedimentos aluviales, transportados por los barrancos que la atraviesan hasta su desembocadura en el mar. Sus límites naturales marcaron el desarrollo de la ciudad, pues hacia el interior se encontraba el obstáculo de las lomas, mientras que hacia el Norte se localizaba un conjunto dunar y un tómbolo, que era invadido periódicamente por las aguas del mar. Por ello, la morfología urbana quedó determinada por la forma de esta franja litoral, que propició un crecimiento longitudinal con una marcada dirección Norte.

En esta franja litoral se localiza, tal y como se ha indicado, la desembocadura de numerosos barrancos. El de mayor superficie es el Guiniguada $\left(65 \mathrm{~km}^{2}\right)$. Su cabecera se sitúa en el interior de la isla, a $1.949 \mathrm{~m}$. La historia de la ciudad ha estado muy ligada a este cauce, 
pues desde su origen, jugó un papel fundamental en la oferta de recursos. Asimismo, podemos distinguir un conjunto de barrancos cuya principal característica es la escasa cuenca de recepción que poseen, inferior a $3 \mathrm{~km}^{2}$. Nacen en las lomas, unidad de paisaje que se describirá más adelante, y son, de Sur a Norte: el Barranco de los Pájaros y el de Osorio, que desembocan en San Cristóbal; el Barranco de Mata, el de Viera (o Lugo) y el de Santa Catalina (o Don Zoilo), que lo hacen en el barrio de los Arenales; el de la Dehesa (o López Socas) y el de las Alcaravaneras, que desembocan en Ciudad Jardín. Gran parte de los problemas que se generan en la ciudad con lluvias intensas están muy ligados a la alteración antrópica de todos estos cauces.

La plataforma litoral limita hacia poniente con un conjunto de lomas, elevaciones que alcanzan entre los 100 y $250 \mathrm{~m}$ de altitud. Esta unidad de paisaje tiene, como rasgo fundamental, su origen sedimentario. Para poder entender la evolución de los materiales que las forman, denominado Formación Detrítica de Las Palmas, es necesario considerar la evolución geológica de la isla, pues en estos sedimentos ha quedado registrada parte de su historia. A grandes rasgos la Formación Detrítica de Las Palmas está compuesta por tres grandes conjuntos, denominados miembros: dos de origen terrestre y uno, intercalado entre ambos, de origen marino. La incisión de los barrancos seccionó los distintos miembros, modelando una serie de interfluvios: son «las lomas», que suavizaron sus formas debido a los procesos de erosión en vertientes.

\section{Metodología y fuentes}

La metodología empleada se inscribe dentro de las propias de los riesgos climáticos (Olcina, 1995) pero adaptada a las particularidades del Archipiélago Canario y al ámbito de estudio: la ciudad de Las Palmas de Gran Canaria. Seguidamente se expone la secuencia metodológica que ha guiado la elaboración de esta investigación así como las fuentes manejadas.

\subsection{Determinación de la secuencia temporal y de su periodización}

Teniendo en cuenta que la investigación pretende relacionar la forma de la ciudad con los efectos de las precipitaciones, la determinación de la secuencia temporal, así como de su periodización, ha estado guiada por la combinación de dos criterios: por una parte, la disponibilidad de datos relativos a las precipitaciones y, por otra, las etapas en las que la forma de la ciudad ha experimentado variaciones significativas.

En cuanto al primer criterio, se opta por iniciar el estudio desde el momento en que comenzaron a efectuarse registros de precipitación de forma continuada en la ciudad, cuestión que sucedió en 1869 con la instalación de la primera estación meteorológica en la ciudad. Por lo que respecta al segundo criterio, se subdividió la secuencia temporal en cuatro etapas (1869-1914; 1915-1949; 1950-1969 y 1970-2000), que responden a las grandes transformaciones sufridas por la trama urbana a lo largo del período considerado.

\subsection{Determinación previa de un umbral de precipitación}

Para la selección de los episodios lluviosos, se establece previamente un umbral de precipitación máxima en 24 horas, basándonos para ello en los intervalos propuestos por Marzol (1988) para Canarias: 
Tabla 1

INTERVALOS DE PRECIPITACIÓN PARA CANARIAS

\begin{tabular}{|c|c|c|c|}
\hline Intervalo $(\mathbf{m m})$ & Calificación & Intervalo $(\mathbf{m m})$ & Calificación \\
\hline $0,1-1,0$ & Lluvia muy débil & $20,1-30,0$ & Lluvia moderada-fuerte \\
$1,1-10,0$ & Lluvia débil & $30,1-50,0$ & Lluvia fuerte \\
$10,1-20,0$ & Lluvia moderada & $+50,0$ & Lluvia muy fuerte \\
\hline
\end{tabular}

FUENTE: Marzol (1988).

Tal y como indica esta autora al clasificar las precipitaciones diarias para el conjunto del Archipiélago Canario, aquéllas que se sitúan en el intervalo comprendido entre los 30,1 y $50,0 \mathrm{~mm}$ se consideran como lluvia fuerte. Dado que el espacio geográfico de estudio es netamente urbano y, generalmente, es en las ciudades donde las precipitaciones diarias, más o menos intensas, ocasionan un mayor número de problemas, se utiliza el límite inferior del umbral considerado como lluvia fuerte para analizar, diacrónicamente, los efectos territoriales y socioeconómicos de estos episodios.

\subsection{Recopilación de datos pluviométricos}

Para conocer la cantidad real de precipitación se dispone de los registros pluviométricos de los observatorios meteorológicos que se fueron ubicando en la ciudad. No obstante, estas estaciones no siempre han estado funcionando de forma continuada a lo largo del período de estudio, ni todas presentan series completas. Así pues, los criterios que han guiado la selección de las estaciones pluviométricas han sido, principalmente, su antigüedad y su ubicación en la trama urbana. En la tabla 2 se exponen los observatorios utilizados, el período en el que estuvieron funcionando y el barrio en el que se ubican.

Tabla 2

ESTACIONES PLUVIOMÉTRICAS SELECCIONADAS Y SU UBICACIÓN POR BARRIOS

\begin{tabular}{|c|c|c|c|c|}
\hline \multirow[b]{2}{*}{ Nombre de la estación } & \multirow[b]{2}{*}{ Localización } & \multirow[b]{2}{*}{ Altitud } & \multicolumn{2}{|c|}{ Periodo } \\
\hline & & & Alta & Baja \\
\hline Instituto de $2^{\mathrm{a}}$ Enseñanza & Vegueta & $31 \mathrm{~m}$ & 1869 & 1875 \\
\hline Colegio de San Agustín & Vegueta & $15 \mathrm{~m}$ & 1882 & 1918 \\
\hline Servicio Agrónomo & San Cristóbal & $12 \mathrm{~m}$ & 1917 & 1936 \\
\hline Puerto de La Luz & Puerto & $15 \mathrm{~m}$ & 1928 & 1994 \\
\hline 003-Las Palmas & Arenales & $15 \mathrm{~m}$ & 1936 & \\
\hline Muelle Deportivo & Alcaravaneras & $10 \mathrm{~m}$ & 1994 & \\
\hline
\end{tabular}

FUENTE: Centro Meteorológico Territorial en Canarias Oriental y Servicio Hidráulico de Las Palmas.

4.4. Caracterización de las consecuencias de los episodios de más de 30,0 mm en el espacio y en la sociedad y fuentes para su estudio

Una vez detectadas todas las situaciones en las que se superó el umbral establecido en alguna de las estaciones seleccionadas, se procedió a su análisis. Para ello se realizó una 
exhaustiva recopilación de información histórica, geográfica y cartográfica para determinar las consecuencias territoriales y socioeconómicas de cada episodio lluvioso.

Las fuentes históricas manejadas son: la prensa, las actas del Ayuntamiento de Las Palmas de Gran Canaria y los informes evacuados por los técnicos municipales tras situaciones de lluvias intensas. De todas ellas es la prensa la que contiene una mayor información, mientras que las restantes tan sólo nos aportan una visión reducida de los efectos de estos episodios. Además son muy contadas las ocasiones en las que aparece alguna reseña en estos dos últimos medios a partir de la segunda mitad del siglo XX.

Por lo que respecta a la prensa, el análisis de esta fuente se ha realizado en un doble sentido. Por un lado se han analizado, diacrónicamente, todos y cada uno de los periódicos existentes desde 1851, fecha de la publicación del primer periódico en la ciudad, hasta la actualidad. Por otro lado, y a partir del umbral establecido de $30,1 \mathrm{~mm}$, se ha recopilado la información existente en todos los periódicos en los episodios en los que se superó esa cantidad. De esta forma se puede establecer un análisis comparativo entre los daños ocasionados en Las Palmas de Gran Canaria como consecuencia de precipitaciones iguales o superiores a 30,1 mm y si existen o no daños con precipitaciones inferiores a esta cantidad. Al mismo tiempo se puede establecer una aproximación en aquellos años en los que, por ausencia de datos pluviométricos al no existir observatorios operativos en la ciudad, se intuye se ha rebasado el umbral.

Por otro lado, las actas del Ayuntamiento de Las Palmas de Gran Canaria muestran todas aquellas disposiciones que la comunidad realiza, cada vez que sucede un acontecimiento excepcional en la ciudad. En el caso que aquí nos ocupa, las referencias ponen de manifiesto los expedientes incoados por particulares que han sufrido daños en sus propiedades, como consecuencia de inundaciones; las medidas de carácter urgente adoptadas para paliar las consecuencias de los desbordamientos de los cauces que atraviesan la ciudad; las peticiones a las autoridades gubernativas para el libramiento de créditos para reparar los daños ocasionados por las lluvias; las valoraciones de los daños producidos por las inundaciones sufridas y su envío a la Delegación del Gobierno, etc.

Finalmente, se han consultado los informes técnicos realizados por el municipio tras un episodio de lluvias intensas. En este caso, el técnico municipal correspondiente elabora informes donde refleja qué áreas se han visto afectadas, las causas que motivaron las inundaciones, el coste que supone la reparación de los daños, y las medidas adoptadas para que en el futuro no se reproduzcan situaciones similares. A diferencia de lo que sucede con otras fuentes como la prensa, los informes realizados por los técnicos municipales detallan minuciosamente la problemática detectada, sobre todo en aquellos casos en que las reparaciones no afectan a grandes áreas y son fácilmente asumibles por la municipalidad. Estos informes, aunque muy escasos, son realmente útiles para determinar las áreas afectadas y los problemas ocasionados tras las lluvias, especialmente en las últimas décadas del siglo XX.

\subsection{Caracterización espacial de las consecuencias de los episodios de lluvia seleccionados y fuentes manejadas}

Para caracterizar espacialmente los problemas ocasionados con las lluvias se procedió al análisis de las distintas unidades ambientales presentes en Las Palmas de Gran Canaria a mediados del siglo XIX, y sus posteriores modificaciones como consecuencia del desarrollo urbano. Ello permite comparar las transformaciones en el medio (la forma 
de ocupación del espacio) y la respuesta de éste cada vez que se producen precipitaciones intensas.

Distintas fuentes históricas, geográficas y cartográficas permiten realizar una aproximación a la forma de la ciudad en cada uno de los períodos. Así, la elaboración de la base cartográfica al finalizar cada uno de éstos permite comparar los dos procesos: expansión de la trama y la localización de los problemas en los episodios superiores a 30,1 mm.

\section{Resultados}

En los 132 años de análisis se constata que en 61 ocasiones se superaron los 30,1 mm de precipitación en 24 horas. No obstante, a esta cifra hay que añadir algunos episodios en los cuales carecemos de datos diarios de precipitación pero en los que, a tenor de las informaciones contenidas en las distintas fuentes consultadas, se debió superar este umbral. Así, en octubre, noviembre y diciembre de 1879 y en los meses de noviembre de 1922, 1925 y 1926, se evidencian cuantiosos problemas en la ciudad.

Por otro lado es significativa la desigual incidencia, en las fuentes consultadas, de noticias o informes que hagan referencia a problemas en la ciudad con motivo de lluvias en los distintos períodos. Así, entre 1869 y 1914 menos de la mitad de las situaciones en las que se superó el umbral de 30,1 mm tienen su reflejo en las fuentes consultadas. En la mayoría de los casos se insertan breves comentarios que aluden al efecto beneficioso de las lluvias para la agricultura, excepto cuando se superan cantidades considerables (más de 50,0 mm diarios), en que los relatos acerca de los daños ocasionados y las áreas afectadas es extenso. Entre 1915 y 1949, ocho de los nueve episodios en los que se superó el umbral establecido tienen un seguimiento importante en las fuentes consultadas referentes a los distintos problemas ocasionados en la ciudad. A partir de 1950 se totalizaron veinticinco episodios en los que se superó los 30,1 mm diarios. En cinco de ellos no aparecen referencias a problemas en la ciudad. Sin embargo, se constata que, en doce casos en los que se recogieron cantidades de lluvia considerablemente inferiores y que varían entre los 10,2 y los 29,1 mm, existen graves daños en la ciudad.

En lo que concierne al origen de estas precipitaciones conviene indicar que, tal y como señalan algunos autores (Marzol, 1988; Font, 1956; Huetz, 1969), tienen su origen en la llegada hasta el Archipiélago de masas de aire polar marítimo, procedentes de las latitudes templadas. Así pues, los principales tipos de tiempo que originan precipitaciones intensas en la ciudad guardan relación con la circulación atmosférica del Oeste de las latitudes medias, y la llegada de borrascas de dirección NE. Para que sea posible la existencia de situaciones de inestabilidad, el anticiclón de las Azores debe haberse retirado de las inmediaciones de Canarias, permitiendo el desplazamiento de borrascas con sus frentes asociados. Por su parte, en las capas medias y altas de la atmósfera se verifica la llegada de masas de aire inestables, con bajas presiones o vaguadas muy pronunciadas. Asimismo, la corriente en chorro «presenta una circulación meridiana con un movimiento ciclónico sobre el Archipiélago. En los episodios de mayor inestabilidad el citado Jet Stream llega a estrangularse y como consecuencia de ello se forma una "gota fría» que puede provocar intensas lluvias generalizadas en toda la isla» (Marzol, 1993: 122).

Así pues, en el origen de las precipitaciones intensas en Canarias cabe señalar, como causa principal, el descenso en latitud de borrascas procedentes del Frente Polar. Dependiendo de las trayectorias seguidas por las masas nubosas que acompañan a estas perturbaciones, su incidencia en el sector NE de Gran Canaria se hará notar con mayor o menor 
intensidad. Si éstas penetran por el S y SE de las isla, las posibilidades de que se registren aguaceros intensos en la ciudad son considerables, no así cuando lo hacen con una marcada dirección $\mathrm{SO}$, en cuyo caso la isla ejerce de barrera orográfica y, por tanto, las áreas del NE reciben escasos aportes de lluvia. Por su parte, si frentes nubosos muy activos penetran en la isla con una dirección N-S, la probabilidad de que se registren lluvias intensas en Las Palmas de Gran Canaria es considerable.

Por lo que respecta a las consecuencias de las precipitaciones intensas en la ciudad se detecta un conjunto de problemas que se repiten en la mayoría de los episodios analizados: desprendimientos, daños en la red viaria y en las edificaciones, caída de paredes y muros de contención, obstrucciones de cloacas y vertidos en la vía pública, etc. No obstante, las inundaciones constituyen el fenómeno que se manifiesta de forma más frecuente en la ciudad. Como se mencionó en la metodología, el estudio de estas consecuencias se ha referido a cuatro períodos que se exponen a continuación.

\section{A) Lluvias e inundaciones entre 1869-1914}

Tal y como se indicó anteriormente, para poder exponer, en un período tan amplio, los daños ocasionados con motivo de las lluvias intensas en la ciudad es preciso señalar, además de las características del medio físico, cuál ha sido su alteración como consecuencia del crecimiento urbano; un crecimiento, tal y como se irá desgranando a lo largo de las siguientes páginas, carente de las adecuadas medidas preventivas en lo que concierne a la evacuación de aguas pluviales o a la necesaria protección frente a los desprendimientos y arrastres de materiales sedimentarios.

En lo que a la ocupación de este espacio se refiere, a mediados del siglo XIX, Las Palmas de Gran Canaria estaba formada fundamentalmente por los núcleos históricos localizados sobre la plataforma litoral, en ambos márgenes del Barranco Guiniguada y en el sector más próximo a su desembocadura: Vegueta y Triana. Los terrenos circundantes a estas áreas estaban destinados al uso agrícola. Asimismo, un conjunto de pobladores sin recursos fueron edificando sus viviendas en las laderas más próximas a la plataforma litoral, junto a Vegueta y Triana; son los denominados Riscos de la ciudad, en alusión al sector topográfico de elevada pendiente que ocupan: San José, San Juan, San Roque, San Nicolás, San Bernardo, San Lázaro y San Antonio.

La expansión de la ciudad en torno a Triana se realizó hacia el Norte, sobre la plataforma litoral, y tiene su origen en el proyecto de apertura de la carretera al Puerto de la Luz, obra iniciada en 1854 y concluida siete años más tarde. El eje de esta vía sirvió como elemento básico de referencia a partir del cual se iban configurando las manzanas sobre las que se parcelaría. Aparece así un nuevo barrio: los Arenales. Años más tarde, en 1890, se aprueba el proyecto de «Apertura de calles en las Huertas de Triana», con el que se urbaniza un importante sector a poniente de este primitivo núcleo.

Hacia finales del siglo XIX, comenzó a desarrollarse una intensa ocupación del espacio en las faldas de la Isleta, al amparo de la construcción del Puerto de La Luz, obra iniciada en la década de los 60 de ese siglo. La localización de esta infraestructura portuaria al pie de una pequeña península, de aproximadamente $8,5 \mathrm{~km}^{2}$, se explica, entre otras causas, por las magníficas condiciones naturales que la existencia de una bahía ofrecía a este tipo de actividades. Una parte de este entorno, alejado del primitivo núcleo de la ciudad, se edificó rápidamente, ocupando el sector más deprimido de la Isleta, así como la ladera Sur más próxima al nuevo puerto. Esta unidad conecta con la plataforma litoral a través 
del tómbolo de Guanarteme, constituido por materiales volcánicos y por sedimentos cuaternarios.

Entre las causas que explican la expansión urbana de este sector destacan el notable crecimiento demográfico de la ciudad junto a una oleada inmigratoria proveniente del campo. Tal y como señala Martín Galán (1984), hacia 1890 proliferaron almacenes, casas y chozas al amparo del proceso urbanizador. Así, hacia 1914, la población de este sector ascendía a 15.000 personas, ubicadas en una trama consolidada al Sur de la Isleta, en el sector más próximo al Puerto.

En este contexto de ocupación, los efectos más importantes de las precipitaciones intensas en los distintos barrios que forman la ciudad hasta 1915 son las inundaciones. Las más graves están motivadas por los desbordamientos del Barranco Guiniguada debido a la invasión de las aguas en las fincas colindantes al cauce, lo que ocasionaba el arrastre de grandes cantidades de piedras, tierra, cultivos, animales, etc. que, a su vez, llegaban a obturar el cauce y represar el agua, generando avenidas mayores. Tal y como se observa en la figura 2, los desbordamientos afectaban principalmente a Vegueta, debido a la rotura de los muros de contención que protegían las huertas y edificaciones del margen derecho del cauce. No obstante, cuando las aguas alcanzaban la altura de los puentes cercanos a la desembocadura, las inundaciones también se producían en Triana.

Por su parte, el Barranco de Mata se desbordó también en varias ocasiones debido a la escasa sección del canal construido para evacuar las aguas de escorrentía, anegando las viviendas más próximas. Otros cauces ubicados al Sur de Vegueta también ocasionaron algunos problemas, especialmente en las huertas de la vega de San Cristóbal, inundando cultivos y obturando pozos y pilares para el abastecimiento de agua potable.

Otros sectores con problemas de inundación son los Riscos. Éstos obedecen a las inadecuadas intervenciones humanas por parte de los numerosos pobladores que se fueron asentando en estas áreas a partir de la década de 1860. Los escasos recursos de estos habitantes, normalmente inmigrantes procedentes del campo, les impedían acceder a las nuevas áreas de expansión de la ciudad, por lo que fueron edificando sus viviendas, normalmente casetas de madera de dimensiones exiguas, por doquier, abriendo veredas, construyendo muros de contención, etc. Estas actuaciones, en un medio de elevada pendiente, ocasionaban el desvío de las aguas de escorrentía hacia las veredas que conducían a las distintas viviendas. Además, los aportes de agua y materiales sedimentarios de las cañadas que descienden por los Riscos eran capaces de inundar y destruir las casas edificadas sobre ellos o en sus proximidades. Las aguas y los materiales arrastrados (lodo, piedras, etc.), procedentes de los riscos de San Nicolás y San Bernardo, se acumulaban en el barrio de Triana anegando numerosas viviendas y establecimientos comerciales (figura 2).

\section{B) Lluvias e inundaciones entre 1914-1949}

Durante este período la ciudad continuó su expansión hacia el Norte por la franja litoral, de tal forma que, hacia mediados del siglo XX, ofrecía el aspecto de un continuo edificado desde Vegueta hasta la Isleta, aunque con espacios intercalados libres de edificaciones. Se asiste así a un proceso de conurbación entre el antiguo casco histórico de la ciudad y el nuevo, nacido al amparo del Puerto de La Luz. Se ocupa así una de las unidades de paisaje más singulares desde el punto de vista ambiental: el istmo de Guanarteme y parte de las playas que se desarrollan a ambos lados de este tómbolo, reflejo de la anti- 


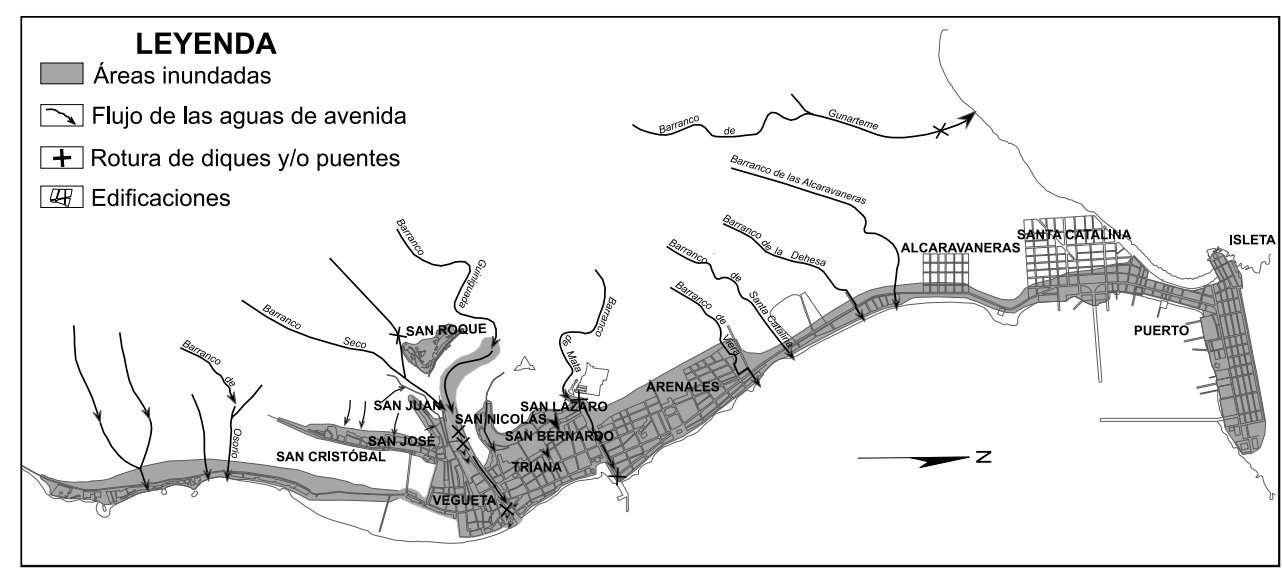

FIGURA 2. Consecuencias de las lluvias diarias superiores a 30,0 $\mathrm{mm}$ en la ciudad de Las Palmas de Gran Canaria (1869-1914).

gua dinámica de circulación de arena: la Playa de las Canteras, hacia poniente, y la de las Alcaravaneras, hacia naciente. Esta circulación de áridos tiene su origen en las corrientes marinas que transportan estos áridos hasta depositarlos en la Playa de Las Canteras. Desde aquí, las arenas eran impulsadas por los vientos alisios hacia el S-SE desapareciendo por la Playa de las Alcaravaneras. Este trasiego de arenas originaba dunas desde el istmo hasta la base más septentrional de las lomas, llegando a ocupar un importante sector de la plataforma litoral. El crecimiento urbano en este sector ocasionó la total ocupación del campo de dunas, apareciendo los barrios de Alcaravaneras, Santa Catalina, Puerto, Canteras y Guanarteme.

Por otro lado, hacia finales de la década de los cuarenta comienza la construcción de viviendas en las lomas de la ciudad, hecho que repercutirá sensiblemente en la aparición de nuevos problemas en la franja litoral. La plataforma de abrasión marina limita con un extenso relieve alomado a través de un pronunciado desnivel, antiguo acantilado marino que establece dos sectores topográficamente diferenciados: la mencionada franja litoral y las lomas. La presencia de acusados desniveles en este área debido a la dinámica de vertientes; la existencia del paleoacantilado en el sector más próximo a la llanura litoral; el carácter heterogéneo y deleznable de la Formación Detrítica, junto con la alteración antrópica de todo este conjunto han ocasionado que, en episodios de lluvias intensas, los aportes de sólidos a las aguas de avenida sean muy elevados. Asimismo, en determinadas áreas de elevada pendiente, el riesgo de desprendimientos es considerable, máxime cuando los materiales han sido removidos para la construcción de vías y edificaciones, cuestión que comienza a ser más evidente a partir de la década de los años 1950.

En lo que concierne a las consecuencias de los episodios de lluvia analizados en este período, éstas no difieren considerablemente de los descritos anteriormente. Así, los desbordamientos de cauces, con las inundaciones asociadas y los daños en edificaciones e infraestructuras agrícolas y ganaderas que pervivían en la ciudad consolidada, continuaron siendo los principales problemas. No obstante, se detectan algunas diferencias con respecto al período anterior, centradas, principalmente, en el espacio afectado por estas inundaciones. 
Prácticamente todos los barrios de la ciudad tienen problemas de inundaciones (figura 3), si bien el más afectado durante este período es el de Arenales. Tanto el área más consolidada y próxima a Triana, como los nuevos crecimientos realizados hacia el Norte, ocupando la práctica totalidad de la franja litoral, fueron áreas anegadizas. Asimismo, el nuevo barrio de Guanarteme, en el extremo occidental de la playa de las Canteras, padeció importantes inundaciones, lo mismo que el Puerto y la Isleta. Barrios como los Riscos, Vegueta y Triana continuaron con los mismos problemas del período anterior.

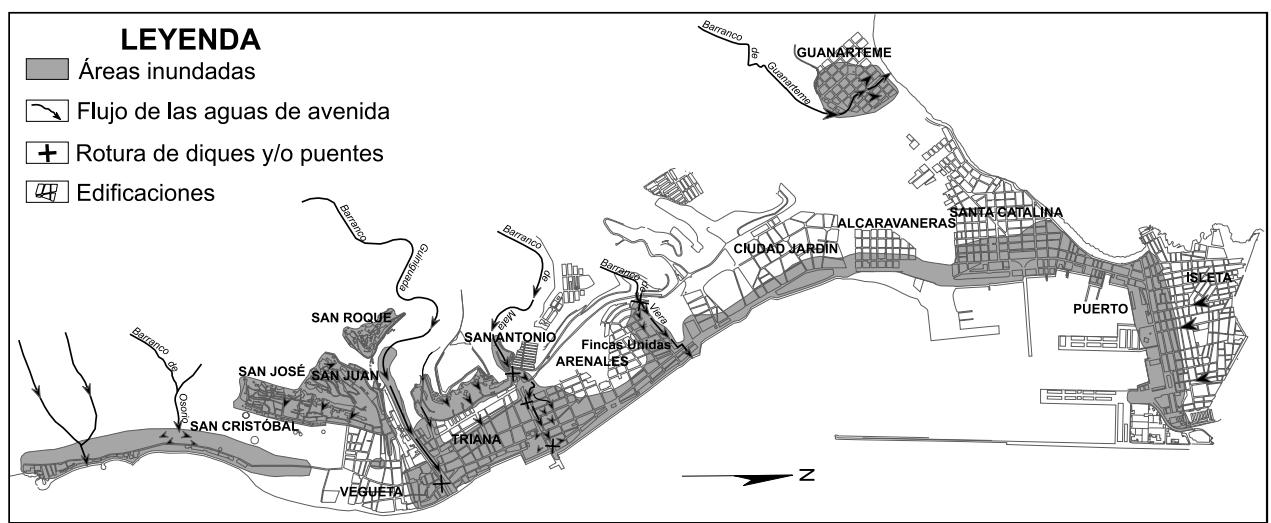

FiguRA 3. Consecuencias de las lluvias diarias superiores a $30,0 \mathrm{~mm}$ en la ciudad de Las Palmas de Gran Canaria (1915-1949).

Por lo que respecta al origen de las inundaciones, siguen siendo los desbordamientos de cauces uno de sus principales motivos. En este sentido, el aumento de las interferencias con éstos, bien por su inadecuada canalización o la escasa sección de los puentes construidos, bien por su desvío o mal estado de conservación, serán el origen de nuevas y graves inundaciones en la ciudad, especialmente en los Arenales, Guanarteme y San Cristóbal. En el caso del Barranco Guiniguada, se detecta una disminución de los problemas asociados al cauce, pues no se constatan incidentes en los canales que evacúan las aguas hasta el mar. Finalmente hay que señalar que el crecimiento de los Riscos fue el origen de nuevas interferencias con los pequeños cauces existentes en estos sectores, lo que motivó la inundación de las viviendas ubicadas en estas áreas junto con otras de la plataforma litoral, especialmente en Triana.

\section{C) Lluvias e inundaciones entre 1950 y 1969}

Uno de los rasgos más característicos de este período es el acelerado crecimiento del perímetro urbano. La iniciativa pública, primero, y la privada, a partir de segunda mitad de la década de los 60 , realizaron múltiples actuaciones tanto en la plataforma litoral como en su orla periférica: Barranco de Mata, lomas de la ciudad y vega de San José. Por lo que respecta a las edificaciones ubicadas en la plataforma litoral se detecta una renovación de las construcciones existentes. Las promociones en este sector tienen como fin principal la sustitución tipológica de edificios y casas, con la asignación de nuevas edifi- 
cabilidades. A esto se añaden las transformaciones experimentadas en determinados sectores, aprovechando la aparición de nuevas funcionalidades: Las Canteras como centro de la industria turística, y el límite más meridional del barrio de Santa Catalina como centro comercial.

Durante este período se amplía artificialmente la plataforma litoral mediante la construcción de un dique sobre el que se construye la Avenida Marítima. La aparición de esta Avenida introduce un cambio sustancial en el sistema natural de evacuación de las aguas pluviales de gran parte de la plataforma litoral. Dispuesta perpendicularmente a la desembocadura de numerosos barrancos, representa un obstáculo difícilmente salvable en momentos de lluvias intensas. Al elevarse 6 ó $7 \mathrm{~m}$ sobre el nivel de la antigua franja costera, genera un sector de encharcamiento constante próximo a la Avenida. Si a esto se añade la escasa dimensión que adquiere la red de alcantarillado al asignar nuevas edificabilidades en los barrios de este sector, se entienden los problemas que surgen con lluvias cada vez más escasas.

Durante los años de este período, la ciudad aumenta considerablemente el espacio urbanizado siguiendo dos vertientes: la legal, mediante numerosas urbanizaciones y polígonos en la orla periférica a la plataforma litoral; y la marginal, principalmente en los Riscos. En ambos espacios se detectan numerosos problemas con las lluvias, aunque es en los Riscos donde son más graves. La crisis de una economía basada en el sector primario ocasionó una fuerte inmigración de población agrícola de poca o nula cualificación profesional, de escasos recursos económicos y, por ello, sin posibilidades para asentarse en las áreas previstas por las normas y planes urbanísticos. Así pues, unas áreas tan transformadas en las décadas precedentes lo serán todavía más durante este período, motivo por el cual concentran el mayor número de víctimas durante los episodios de lluvia. Siete de las ocho que hubo a lo largo de estos años lo fueron como consecuencia de la caída de muros de contención sobre sus viviendas, reflejo de las pésimas condiciones de vida de estos pobladores.

Por lo que respecta a las consecuencias de los episodios de lluvia intensa, además de los indicados anteriormente, hay que señalar, nuevamente, los problemas ocasionados por las inundaciones en distintos barrios de la ciudad. Los ubicados en la plataforma litoral padecieron las más graves por diversos motivos: la alteración de las cuencas de los pequeños barrancos cuyas cabeceras se localizan en las lomas de la ciudad, el vertido de los escombros procedentes de las urbanizaciones y polígonos en construcción en sus cabeceras, o la ocupación de los cauces para localizar las vías de comunicación; la ausencia, en unos casos, o la escasa dimensión, en otros, de elementos de evacuación de aguas pluviales; la construcción, de la Avenida Marítima; y, finalmente, el aumento de la edificabilidad, lo cual ocasiona mayores aportes a la red unitaria de saneamiento y, por consiguiente, su incapacidad para evacuar los caudales suplementarios de las precipitaciones.

Otros barrios con problemas de inundación son los ubicados en la Isleta, en el istmo y en la playa de Las Canteras. En el primer sector aparecen abundantes referencias en la prensa que aluden a las inundaciones de las vías y viviendas. Las aguas procedentes de las laderas de la Isleta, ahora impermeabilizadas por el desarrollo urbano, desembocan en los sectores más deprimidos y próximos al Puerto. La incapacidad de los elementos de evacuación de aguas pluviales ocasiona la inundación de este sector. Además, determinadas áreas próximas a Las Canteras y Guanarteme también se inundan debido a la incapacidad de la red de alcantarillado para evacuar los aportes provenientes de las lluvias. 


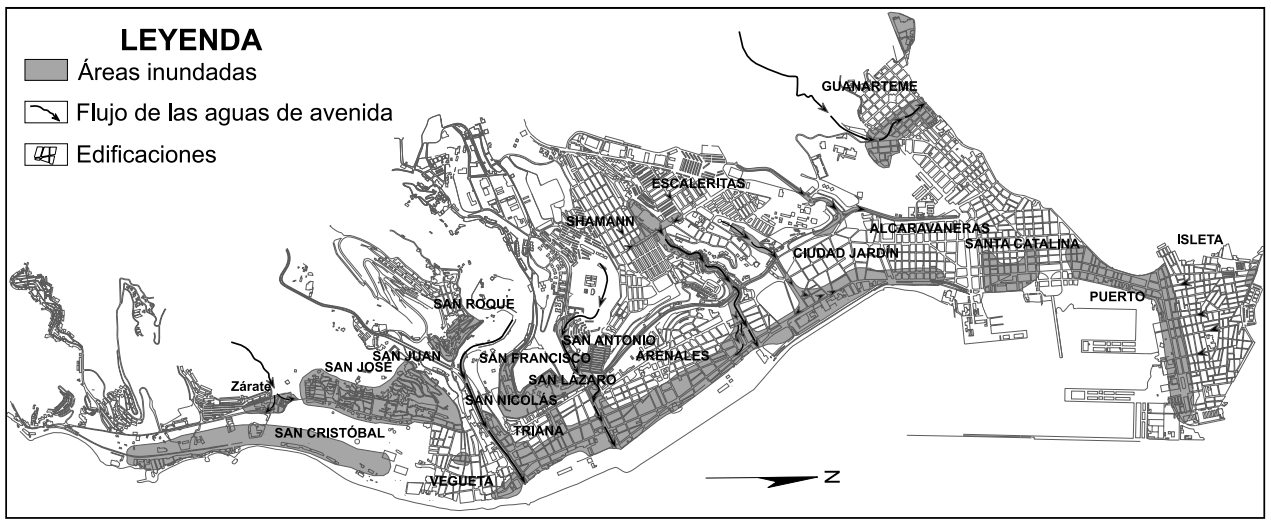

Figura 4. Consecuencias de las lluvias diarias superiores a 30,0 $\mathrm{mm}$ en la ciudad de Las Palmas de Gran Canaria (1950-1969).

\section{D) Lluvias e inundaciones entre 1970 y 2000}

Durante estos últimos años la ciudad ha visto nacer un conjunto de actuaciones tendentes a la creación de grandes espacios urbanos residenciales, la regeneración de otros ya implantados, y la dotación de grandes equipamientos comerciales, educativos, sanitarios y de esparcimiento y ocio. La regeneración de espacios residenciales consolidados se ha llevado a cabo en diversos sectores de los Arenales y el Puerto. El proceso de nueva implantación en áreas poco aptas por su elevada pendiente, principalmente en todo el sector que comprende el paleoacantilado y las laderas de las lomas de la ciudad, motiva la aparición de desprendimientos durante y después de las lluvias.

Por su parte, en las lomas de la ciudad se ha procedido a colmatar el suelo disponible y, sobre todo, a dotar este área con numerosos equipamientos comerciales. Por ello, durante este período, se produce la total desaparición de las pequeñas cuencas que se desarrollaban en este sector, siendo difícil hoy identificar los elementos «naturales» que los componían, convertidos en una mera agregación de muros de contención, vías y casas. Las aguas procedentes de las precipitaciones se evacúan a través de una red de alcantarillado que es incapaz de contener los aportes de las precipitaciones. Los caudales se concentran en tiempos excesivamente cortos debido a la impermeabilización de todo este sector, lo cual impide su evacuación por los elementos de recogida de las aguas pluviales. Al mismo tiempo, la insuficiente sección de la red de alcantarillado ocasiona el vertido de las aguas residuales y pluviales a las vías públicas y sótanos de las viviendas.

Durante este período también quedan finalizados numerosos polígonos y urbanizaciones iniciados a finales de la década de los sesenta. Éstos se localizan en el sector más meridional de la plataforma litoral, al Sur del barrio de San José y en San Cristóbal. Igualmente, nuevas urbanizaciones aparecen en la cuenca del Barranco de Mata, y en la periferia de la ciudad. Muchas de estas actuaciones se caracterizan por la necesidad de levantar grandes muros de contención al estar localizadas en áreas de elevada pendiente. Además, se dotan de elementos para la evacuación de aguas pluviales y residuales de forma separada, mediante redes suficientemente dimensionadas. No obstante, el peculiar crecimiento de la ciudad, 
mediante meras agregaciones de tramas urbanas, facilita que las conducciones queden rápidamente obsoletas cuando otras edificaciones posteriores conectan sus redes a las existentes, motivo por el cual son incapaces de evacuar los nuevos aportes.

Por lo tanto, las nuevas áreas se convierten en nuevos sectores anegadizos (figura 5). Surgen así inundaciones en polígonos como pueden ser Zárate, Hoya de la Plata, Pedro Hidalgo y San Cristóbal, en el sector más meridional de la ciudad; Miller Bajo, Las Rehoyas o el Polvorín, en la cuenca del Barranco de Mata; Escaleritas y Schamann, en la cuencas del Barranco de Don Zoilo; y La Paterna en la periferia de la ciudad.

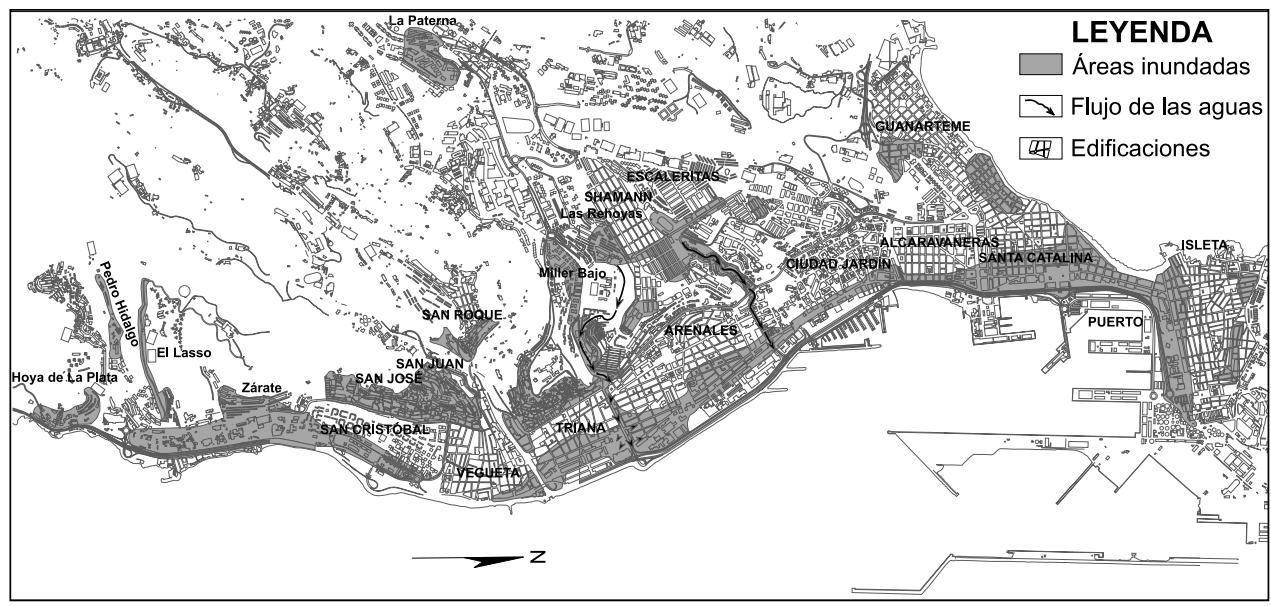

Figura 5. Consecuencias de las lluvias diarias superiores a $30,0 \mathrm{~mm}$ en la ciudad de Las Palmas de Gran Canaria (1970-2000).

\section{Conclusiones}

Los problemas derivados de las precipitaciones intensas en Las Palmas de Gran Canaria experimentan un traslado progresivo hacia las nuevas áreas de crecimiento. Mientras la ciudad se expande hacia el $\mathrm{N}$ a través de la plataforma litoral, la alteración de los cauces en este sector origina gran parte de las inundaciones. A medida que el proceso urbanizador se extiende por las lomas de la ciudad, las intervenciones en las cuencas de todos estos barrancos (vertidos de escombros, ocupación de cauces para viales y su desvío, etc.) originan inundaciones en barrios topográficamente más bajos como Triana, Arenales y Ciudad Jardín. Sin embargo, a medida que el proceso urbanizador ha terminado por ocultarlos bajo vías y edificios es difícil discernir si el motivo de las inundaciones es la insuficiente canalización de los mismos o las deficiencias de la red de saneamiento.

En este sentido, es precisamente la existencia de una red unitaria de saneamiento, incapaz de evacuar los aportes de pluviales y sólidos provenientes tanto de la Formación Detrítica de Las Palmas como de actividades humanas, una de las principales causas de inundaciones en la ciudad. La ausencia histórica, en los documentos de planificación, de medidas para la evacuación de aguas pluviales es otra de las causas que genera cuantiosos problemas en los distintos barrios de la ciudad. Así, cada vez que se producen inundaciones, se intentan resolver a posteriori, y no mediante un proceso meditado de planificación y pre- 
vención. Si a ello se añade las peculiares características del proceso urbanizador en la ciudad, con un crecimiento en el que no ha existido una visión de conjunto para la ciudad, con grandes carencias en infraestructuras, se entiende la incapacidad de la red de saneamiento a medida que se incrementan los caudales de residuales y pluviales provenientes tanto de las nuevas áreas de crecimiento, como de áreas consolidadas a las que se les ha asignado mayores edificabilidades.

Finalmente, y en lo que concierne al umbral de precipitación escogido y su relación con las fuentes históricas manejadas conviene indicar lo siguiente: el umbral de 30,1 $\mathrm{mm}$ no sería válido si queremos analizar las consecuencias de estas lluvias con anterioridad a 1900, pues no existen referencias en documentos históricos que hagan alusión a problemas en la ciudad. En fechas posteriores y hasta 1950, se ajusta bastante bien pues más del $80 \%$ de los casos tiene un correlato informativo de daños en la ciudad en las fuentes manejadas. El caso contrario, esto es, con precipitaciones inferiores a esta cantidad, también encuentra sustanciosas referencias de inundaciones y daños a partir de la década de 1960, lo cual evidencia, entre otras cosas, que el problema es cada vez más acuciante y, por ende, más difícil de solucionar por los costes que conlleva las medidas estructurales que, ahora, han empezado a acometerse.

\section{Bibliografía}

FONT TULLOT, I. (1956): El tiempo atmosférico en las Islas Canarias. Servicio Meteorológico Nacional. Serie A (memorias n⿳⺈ 26), Madrid.

HUETZ DE LEMPS, A. (1969): Le climat de Iles Canaries. París, S.E.D.E.S.

MARTÍN GALÁN, F. (1984): La formación de Las Palmas: Ciudad y Puerto. Cinco siglos de evolución. Santa Cruz de Tenerife. Junta del Puerto de la Luz y de Las Palmas, Gobierno de Canarias, Cabildo Insular de Gran Canaria y Ayuntamiento de Las Palmas de Gran Canaria.

MARZOL, M.V. (1988): La lluvia, un recurso natural para Canarias. Santa Cruz de Tenerife. Caja General de Ahorro de Canarias.

MARZOL, M.V. (1993): El clima: rasgos generales. En Geografía de Canarias. Tomo I: 101-116. Las Palmas de Gran Canaria, Prensa Canaria Ibérica S.A.

OLCINA, J. (1995): Factor climático y la ordenación del territorio: los riesgos climáticos. Situaciones de riesgo climático en España. I.E.P. pp. 15-69.

ORTEGA, F. (1991): Incertidumbre y riesgos naturales. XII Congreso Nacional de Geografía. A.G.E. pp. 101-108. 